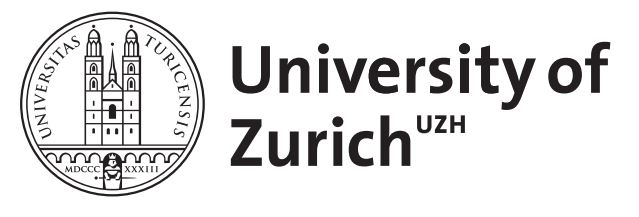

\title{
Epigenetik bei entzündlichen Systemerkrankungen
}

\author{
Ospelt, C ; Gay, S
}

\begin{abstract}
In addition to analysis of the genetic code, in recent years more and more studies have concentrated on changes in the epigenetic code. Epigenetic mechanisms determine which genes in a cell are transcribed and thus form the phenotype of a cell. The epigenetic code can be changed by environmental influences, which allows cells to adapt to longstanding changes in the environment. Therefore, it is feasible to assume that epigenetic changes are the molecular basis for long-term effects of the environment on disease development. In particular in tumors and chronic inflammatory diseases epigenetic changes were found to correlate with disease severity and progression. Knowledge about these epigenetic changes might help that epigenetic modifications can be used in the future as biomarkers, prognostic factors and therapeutic targets.
\end{abstract}

DOI: https://doi.org/10.1007/s00108-013-3373-x

Other titles: Epigenetics in inflammatory systemic diseases

Posted at the Zurich Open Repository and Archive, University of Zurich

ZORA URL: https://doi.org/10.5167/uzh-97634

Journal Article

Published Version

Originally published at:

Ospelt, C; Gay, S (2014). Epigenetik bei entzündlichen Systemerkrankungen. Der Internist, 55(2):124127.

DOI: https://doi.org/10.1007/s00108-013-3373-x 
Internist 2014 · 55:124-127

DOI 10.1007/s00108-013-3373-x

Online publiziert: 9. Januar 2014

๑) Springer-Verlag Berlin Heidelberg 2014

\section{Schwerpunktherausgeber}

W.L. Gross, Lübeck

S. Schreiber, Kiel

\section{Ospelt · S. Gay}

Zentrum für Experimentelle Rheumatologie, Klinik für Rheumatologie, UniversitätsSpital Zürich, Schweiz
In den letzten Jahren konnten mithilfe genomweiter Assoziationsstudien (GWAS) immer mehr „single nucleotide polymorphisms“ (SNP) mit verschiedenen Erkrankungen assoziiert werden. Trotzdem können beispielsweise bei der rheumatoiden Arthritis (RA) die bis jetzt gefundenen SNP nur etwas mehr als ein Drittel der Erblichkeit erklären [10]. Epigenetische Veränderungen haben einen tiefgreifenden, stabilen Einfluss auf die Genexpression, ohne dabei die Sequenz der DNA zu verändern. Wenigstens zum Teil sind sie auch an die Nachkommen vererbbar. Während die DNA-Sequenz den Genotyp definiert und in jeder Zelle eines Individuums prinzipiell gleich ist, regulieren epigenetische Mechanismen, welche Gene in welcher Zelle transkribiert werden. Damit bestimmen sie den Phänotyp einer Zelle. Im Gegensatz zum genetischen Code, der sich in der Regel im Laufe des Lebens nicht verändert, hat der epigenetische Code eine gewisse Plastizität und kann durch Umwelteinflüsse modifiziert werden. Dieser Mechanismus ermöglicht den Zellen, sich an lang anhaltende Veränderungen in ihrer Umgebung anzupassen. Er erinnert an die Evolutionstheorie von Lamarck, die besagt, dass auch Fähigkeiten, die im Laufe des Lebens erworben wurden, vererbt werden können.

\section{Der epigenetische Code}

Die DNA bildet im Kern zusammen mit den Histonen, um die sie gewickelt ist, das Chromatin. Epigenetische Modifikationen können direkt an der DNA oder aber an den frei stehenden Histonseitenketten, den sog. Histonarmen, platziert sein (• Abb. 1). Die Modifikationen der Aminosäuren der Histonarme sind vielfältig und umfassen u. a. Acetylierungen, Methylierungen und Phosphorylierungen. Modifikationen der Histonseitenketten können durch ihre Ladung zu einer Auflockerung des Chromatins und damit zu einer besseren Zugänglichkeit der DNA für Transkriptionsfaktoren führen. Häufiger jedoch dienen sie als Bindungsstelle für Effektorproteine, die wiederum die Transkriptionsmaschinerie stabilisieren oder hemmen können.

Im Gegensatz dazu ist die Methylierung bzw. Hydroxymethylierung von Cytosin bislang die einzige bekannte epigenetische Modifikation der DNA. Die Demethylierung der Cytosine in der Promotorregion eines Gens ermöglicht dessen Transkription, während die Methylierung eines Promotors zur Abschaltung des Gens führt. Die Gesamtheit der epigenetischen Veränderungen an einem bestimmten Gen ist der epigenetische Code, der je nach Zusammensetzung die Expression des Gens blockiert oder zulässt.

\section{》) Auch chronisch- inflammatorische Erkrankungen gehen mit epigenetischen Veränderungen einher}

Veränderungen im epigenetischen Code wurden zuerst in Tumorzellen analysiert. Die Hypermethylierung von Tumorsuppressorgenen und die damit einhergehende Blockade der Expression sowie die aberrante Expression von Onkogenen durch Verlust der Methylierung sind verbreitete Mechanismen, die zur malignen Transfor- mation führen und in den verschiedensten Tumortypen gefunden wurden [9].

Auch im Zusammenhang mit chronisch-inflammatorischen Erkrankungen zeigen immer mehr Studien, dass epigenetische Veränderungen mit dem Krankheitsverlauf einhergehen. Im rheumatologischen Formenkreis wurden sowohl beim systemischen Lupus erythematodes als auch bei der RA und systemischen Sklerose von Gesunden abweichende Muster der DNA-Methylierung und Histonmodifikationen gefunden [7]. So ist beispielsweise in synovialen Fibroblasten von RA-Patienten der Promotor des Chemokins CXCL12 demethyliert, was $\mathrm{zu}$ einer hohen basalen Expression dieses proinflammatorischen Faktors führt [6]. Es wird vermutet, dass dieser oder ähnliche Mechanismen zur Chronifizierung von Entzündungen führen.

\section{MicroRNA}

Neben epigenetischen Modifikationen der Histonarme und der DNA beeinflussen auch nichtcodierende RNA-Moleküle die Genexpression substanziell, $u$. a. lange nichtcodierende RNAs (lncRNA) und microRNAs. Die Funktion der lncRNAs ist großenteils ungeklärt. Es wird angenommen, dass sie Enzyme, die epigenetische Modifikationen katalysieren, sequenzspezifisch an den richtigen Ort im Chromatin führen. MicroRNAs binden an Messenger-RNA (mRNA) und können dadurch deren Umschreibung in Proteine hemmen.

Die Expression von microRNAs ist bei vielen Erkrankungen dereguliert. 
Hier steht eine Anzeige.

黛 Springer 


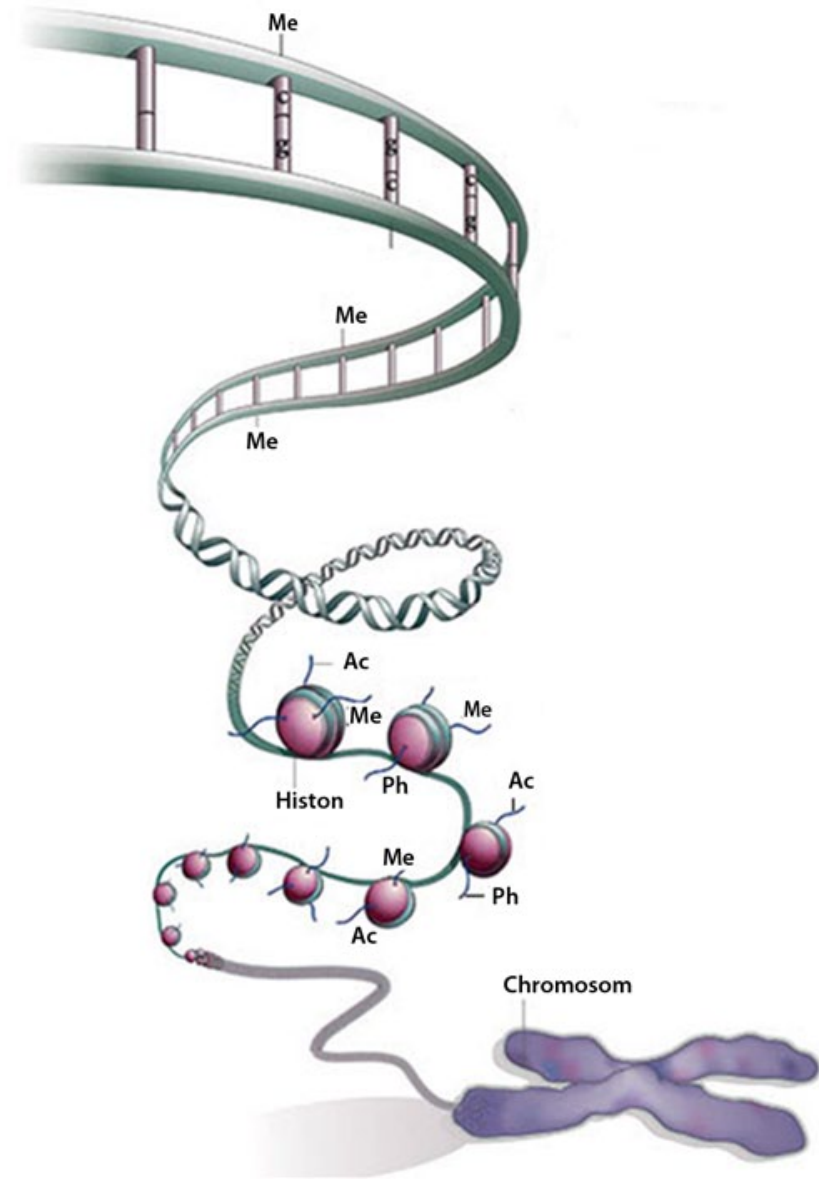

Abb. $1<$ Der epigenetische Code. Sowohl die DNA als auch die Histonarme können epigenetische Modifikationen tragen, die in ihrer Gesamtheit den epigenetischen Code ausmachen. Me Methylierung, Ac Acetylierung, Ph Phosphorylierung. (Adaptiert nach [12])
Da microRNAs auch im Serum sehr stabil sind, könnten sie die nächste Generation von Biomarkern werden [3]. So ließ sich etwa zeigen, dass das Auftreten der leberspezifischen microRNA miR-122 im Blut gut mit Acetaminophen-induzierten Leberschäden korreliert [8]. Bei RA-Patienten korrelierten hohe miR-16-Plasmawerte zu Beginn der Therapie mit einem besseren Ansprechen und einer stärkeren Verminderung der Krankheitsaktivität [2].

\section{Genetik und Epigenetik}

Wie bereits erwähnt kann der epigenetische Code von Umwelteinflüssen moduliert und vererbt werden. Sehr eindrücklich konnte dies an Mäusen gezeigt werden, deren Mütter während der Tragezeit mit methylierenden Nahrungsergänzungsmitteln gefüttert wurden. Durch die spezielle Nahrung der Mütter wurde in den Nachkommen das Gen für die helle Fellfarbe methyliert, was zu einer dunklen Färbung der Jungtiere führte [11]. Auch bei Menschen wurde ein Einfluss der Nahrung auf die DNA-Methylierung gefunden. Bei Kindern, deren Mütter während des niederländischen Hungerwinters 1944/1945 schwanger waren, war das Gen des ,insulin-like growth factor II“ hypomethyliert [5]. Dies könnte der Grund für das häufigere Auftreten von Typ-2-Diabetes und Adipositas bei diesen Nachkommen sein.

Aber auch der genetische Code beeinflusst das Epigenom. SNP in microRNAs können beispielsweise dazu führen, dass die microRNA ihre Ziel-mRNA nicht mehr erkennt und damit auch nicht mehr inhibiert. Andererseits kann ein SNP in der Zielsequenz die Bindung einer microRNA oder auch einer lncRNA verhindern. So zerstört etwa eine stille Mutation im IRGM-Gen die Bindungsstelle der microRNA miR-196. Bei einer Entzündung der Darmmukosa wird miR-196 induziert, was normalerweise zu einer Reduktion des IRGM-Proteins führt. Verminderte IRGM-Spiegel sind wiederum die Voraussetzung für die Beseitigung von Mor-
bus-Crohn-assoziierten, adhärent-invasiven Escherichia-coli-Stämmen [1]. Da Individuen mit dem Risiko-SNP im IRGMGen ein erhöhtes Risiko haben, an Morbus Crohn zu erkranken, liegt die Vermutung nahe, dass der Krankheitsausbruch mit der Persistenz adhärent-invasiver E. coli zusammenhängt.

\section{Epigenetik in der Praxis}

Auch wenn viele epigenetische Studien heute an Zellen oder Tiermodellen durchgeführt werden, wird eine Nutzung des Wissens um epigenetische Veränderungen in der Praxis voraussichtlich möglich sein, sowohl in Form von Biomarkern und prognostischen Faktoren wie auch als therapeutischer Ansatz. Der Einsatz als Biomarker bietet sich v. a. für microRNAs an.

Therapeutisch werden große Hoffnungen in die Hemmung histonmodifizierender Enzyme gesetzt. Hemmer von Histondeacetylasen (HDACi) sind für die Behandlung von Lymphomen zugelassen und zeigten in Tiermodellen für RA, Kolitis, multiple Sklerose und Lupus eine gute antiinflammatorische Wirkung [4].

\section{\) Hemmer von \\ Histondeacetylasen zeigten \\ in Tiermodellen eine gute antiinflammatorische Wirkung}

In der Behandlung von RA und chronisch-entzündlichen Darmerkrankungen verdichten sich die Beweise, dass in einem frühen Stadium behandelte Patienten gröBere Chancen auf eine Remission haben. Ein epigenetischer Hintergrund dieses Gelegenheitsfensters ist denkbar. Vermutlich werden bei chronisch-entzündlichen Erkrankungen nach lang anhaltender Krankheitsaktivität die entzündlichen Signalwege epigenetisch modifiziert, sodass sie stabil aktiviert bleiben und nicht mehr ausgeschaltet werden können. In frühen Krankheitsstadien sind die Zellen noch nicht geprägt, ihre Funktion kann somit leichter wieder normalisiert werden. Sollte sich diese Theorie bewahrheiten, könnten viele chronische Krankheiten mit Medikamenten kuriert werden, die das Epigenom normalisieren. 


\section{Fazit für die Praxis}

- Der epigenetische Code kann durch Umwelteinflüsse modifiziert werden. Aber auch der genetische Code beeinflusst das Epigenom, so etwa „,single nucleotide polymorphisms" in microRNAs.

- Modifikationen der Histonseitenketten können eine bessere Zugänglichkeit der DNA für Transkriptionsfaktoren bewirken. Häufiger jedoch dienen sie als Bindungsstelle für Effektorproteine, die wiederum die Transkriptionsmaschinerie stabilisieren oder hemmen können.

- Die Demethylierung der Cytosine in der Promotorregion eines Gens ermöglicht dessen Transkription, die Methylierung führt zur Abschaltung des Gens.

- Bei verschiedenen rheumatologischen Erkrankungen wurden von Gesunden abweichende Muster der DNA-Methylierung und Histonmodifikationen gefunden.

- MicroRNAs könnten die nächste Generation von Biomarkern werden.

- Therapeutisch setzt man große Hoffnungen in die Hemmung histonmodifizierender Enzyme.

\section{Korrespondenzadresse}

C. Ospelt
Zentrum für Experimentelle
Rheumatologie,
Klinik für Rheumatologie,
UniversitätsSpital Zürich
Gloriastr. 23, 8091 Zürich
Schweiz
caroline.ospelt@usz.ch

\section{Einhaltung ethischer Richtlinien}

Interessenkonflikt. C. Ospelt und S. Gay geben an, dass kein Interessenkonflikt besteht.

Dieser Beitrag beinhaltet keine Studien an Menschen oder Tieren.

\section{Literatur}

1. Brest P, Lapaquette P, Souidi M et al (2011) A synonymous variant in IRGM alters a binding site for miR-196 and causes deregulation of IRGM-dependent xenophagy in Crohn's disease. Nat Genet 43:242-245

Internist 2014 - 55:124-127 DOI 10.1007/s00108-013-3373-X

C Springer-Verlag Berlin Heidelberg 2014

C. Ospelt · S. Gay

\section{Epigenetik bei entzündlichen Systemerkrankungen}

Zusammenfassung

In den letzten Jahren wurden neben genetischen Studien immer mehr Analysen von Veränderungen im epigenetischen Code durchgeführt. Epigenetische Mechanismen bestimmen, welche Gene in welcher Zelle transkribiert werden. Damit formen sie den Phänotyp. Der epigenetische Code kann durch Umwelteinflüsse modifiziert werden. Dies ermöglicht den Zellen, sich an lang anhaltende Veränderungen in ihrer Umgebung anzupassen. Daher liegt der Schluss nahe, dass epigenetische Veränderungen die Grundlage für langfristige Auswirkungen von Umwelteinflüssen auf die Gesundheit sein kön- nen. Vor allem bei Tumoren und chronischentzündlichen Erkrankungen zeigen immer mehr Studien, dass mit dem Krankheitsverlauf epigenetische Veränderungen einhergehen. Das Wissen um epigenetische Veränderungen könnte zukünftig in der Praxis genutzt werden, sowohl als Biomarker und prognostischer Faktor wie auch als therapeutischer Ansatz.

Schlüsselwörter

MicroRNA · Chronische Entzündungen .

DNA-Methylierung · Histonmodifikationen . Biomarker

\section{Epigenetics in inflammatory systemic diseases}

\section{Abstract}

In addition to analysis of the genetic code, in recent years more and more studies have concentrated on changes in the epigenetic code. Epigenetic mechanisms determine which genes in a cell are transcribed and thus form the phenotype of a cell. The epigenetic code can be changed by environmental influences, which allows cells to adapt to longstanding changes in the environment. Therefore, it is feasible to assume that epigenetic changes are the molecular basis for longterm effects of the environment on disease development. In particular in tumors and chronic inflammatory diseases epigenetic changes were found to correlate with disease severity and progression. Knowledge about these epigenetic changes might help that epigenetic modifications can be used in the future as biomarkers, prognostic factors and therapeutic targets.

Keywords

MicroRNA · Chronic inflammation · DNA methylation $\cdot$ Histone modifications . Biomarker
2. Filkova M, Aradi B, Senolt L et al (2013) Association of circulating miR-223 and miR-16 with disease activity in patients with early rheumatoid arthritis. Ann Rheum Dis (im Druck)

3. Filkova M, Jungel A, Gay RE et al (2012) MicroRNAs in rheumatoid arthritis: potential role in diagnosis and therapy. BioDrugs 26:131-141

4. Hancock WW, Akimova T, Beier UH et al (2012) HDAC inhibitor therapy in autoimmunity and transplantation. Ann Rheum Dis 71(Suppl 2):i46i54

5. Heijmans BT, Tobi EW, Stein AD et al (2008) Persistent epigenetic differences associated with prenatal exposure to famine in humans. Proc Natl Acad Sci U S A 105:17046-17049

6. Karouzakis $E$, Rengel $Y$, Jungel A et al (2011) DNA methylation regulates the expression of CXCL12 in rheumatoid arthritis synovial fibroblasts. Genes Immun 12:643-652

7. Ospelt C, Gay S (2013) Epigenetics. In: Firestein GS Budd RC, Gabriel SE, McInnes I, O’Dell JR (Hrsg) Kelley's textbook of rheumatology. Elsevier, Philadelphia, S 316-325

8. Shi Q, Yang X, Mendrick DL (2013) Hopes and challenges in using miRNAs as translational biomarkers for drug-induced liver injury. Biomark Med 7:307-315
9. Suva ML, Riggi N, Bernstein BE (2013) Epigenetic reprogramming in cancer. Science 339:1567-1570

10. Viatte S, Plant D, Raychaudhuri S (2013) Genetics and epigenetics of rheumatoid arthritis. Nat Rev Rheumatol 9:141-153

11. Waterland RA, Jirtle RL (2003) Transposable elements: targets for early nutritional effects on epigenetic gene regulation. Mol Cell Biol 23:52935300

12. Qiu J (2006) Epigenetics: unfinished symphony. Nature 441:143-145

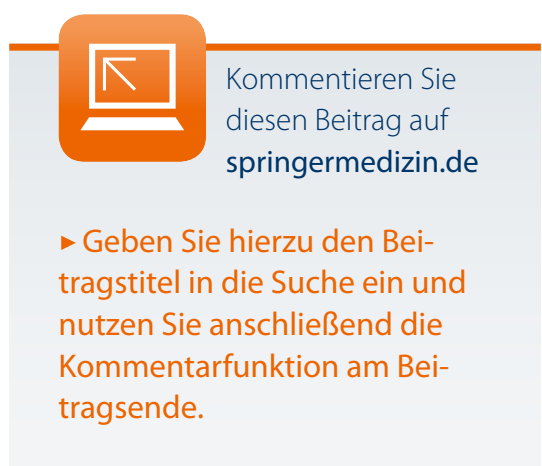

